\title{
Computational Properties of Peri-Dendritic Calcium Fluctuations
}

\author{
David M. Egelman and P. Read Montague \\ Division of Neuroscience, Center for Theoretical Neuroscience, Baylor College of Medicine, Houston, Texas 77030
}

\begin{abstract}
Using a model of the extracellular space, we show how external calcium fluctuations, engendered during normal neural activity, can act as a rapid information-bearing signal in nervous systems. We demonstrate that action potentials propagating along a dendrite can induce large peri-dendritic calcium fluctuations, lowering significantly the external calcium available to overlying presynaptic terminals. The geometrical distribution of active calcium sinks critically influences the time and spatial extent of fluctuations in external calcium. In particular, clusters of coactive dendrites can prolong and amplify an external calcium
\end{abstract}

The calcium ion is involved in many signaling pathways and is necessary for synaptic transmission and plasticity (Dodge and Rahamimoff, 1967; Katz and Miledi, 1970; Bootman and Berridge, 1995; Mintz et al., 1995; Qian et al., 1997). To date, intracellular signaling pathways involving calcium have received the most detailed experimental examination, leaving virtually unexplored the potential roles played by fluctuations in extracellular calcium. It has long been known from electrode measurements that synaptic activation can cause large (on the order of millimolar) changes in external calcium (Nicholson et al., 1978). Only recently has it been appreciated that these fluctuations may represent an information-bearing signal to nearby neural elements (Smith, 1992; Montague, 1996; Egelman et al., 1998).

We focus here on the fact that neurons produce action potentials that propagate into their axons and dendrites (Stuart and Sakmann, 1994). Action potentials that invade the dendrites of a cell cause large fluxes of calcium into the dendrite through a variety of voltage-gated calcium channels (Magee and Johnston, 1995; Yuste and Tank, 1996). This influx of calcium is mirrored by an efflux of calcium from the extracellular space just outside the dendrite. Hence, the timing of the back-propagating spike (BP-spike) can be encoded briefly in a fluctuation in external calcium in the peri-dendritic region (Fig. 1).

As we will demonstrate below, external calcium levels and the geometry of the extracellular space are arranged so that neural activity can cause significant fluctuations in external calcium. The potential significance of such fluctuations derives from the many roles that calcium plays in synaptic transmission, long-term synaptic modulation, cell adhesion, growth cone motility, and a vast number of intracellular signaling pathways. External calcium

\footnotetext{
Received July 16, 1998; accepted Aug. 10, 1998.

This work was supported by the Center for Theoretical Neuroscience at Baylor College of Medicine and by National Institute of Mental Health Grant RO1 MH52797. We thank Drs. Francis Crick, Peter Dayan, Dan Johnston, Thomas Bartol, and Saurabh Sinha for helpful comments before the preparation of this manuscript. Help in many aspects is gratefully acknowledged to Richard King.

Correspondence should be addressed to Dr. P. Read Montague, Center for Theoretical Neuroscience, Baylor College of Medicine, One Baylor Plaza, Houston, TX 77030.

Copyright (ㄷ) 1998 Society for Neuroscience $\quad 0270-6474 / 98 / 188580-10 \$ 05.00 / 0$
}

fluctuation. This latter effect provides a natural substrate for a computational mechanism that locates specific volumes of neural tissue on rapid time scales. Such an interpretation suggests that the detailed structure of the extracellular space, in combination with the three-dimensional distribution of active calcium sinks, may play a role in neural information processing.

Key words: resource consumption principle; backpropagating action potential; self-organized computing; dendrites; content-addressable memory; external calcium

fluctuations can be studied from two basic perspectives: biophysical and computational. At the biophysical level, one can appeal to the physical mechanisms used by neural elements to construct, detect, and respond to external calcium fluctuations. Using a simplification of the biophysical problem, we focus instead on the potential computational roles of these fluctuations. We pursue the hypothesis that external calcium fluctuations engendered by normal neural activity represent an information-bearing signal. In particular, we show how patterns of synaptic activation in a broad region of neural tissue could use external calcium fluctuations in a mechanism that rapidly locates small volumes of neural tissue.

To calculate external calcium fluctuations, we have developed a finite-difference model of the extracellular space and calibrated it using a combination of analytic and Monte Carlo methods (see Materials and Methods). Our results are presented in three parts. (1) We demonstrate that back-propagating action potentials can induce large peri-dendritic calcium fluctuations, thereby lowering the calcium available to overlying presynaptic terminals; (2) we show how different geometrical arrangements of dendrites, dendritic spines, and calcium sinks can act to change the amplitude and time course of external calcium fluctuations; and (3) we describe how these calcium fluctuations could be part of a mechanism that rapidly indexes specific volumes of neural tissue.

\section{MATERIALS AND METHODS}

Strategy. Monte Carlo calculations and the finite-difference model of the extracellular space were programmed in $\mathrm{C}$ and run on Silicon Graphics workstations. To calibrate the finite-difference model, we calibrated the Monte Carlo simulator first against free diffusion and then introduced the reflecting boundaries. The Monte Carlo model was then used to calibrate the finite-difference model of the extracellular space, which runs three to four orders of magnitude faster. In both models, the membranes of synapses, spines, and dendrites were implemented explicitly as reflecting boundaries.

Monte Carlo calculations. In the Monte Carlo simulator, external calcium was implemented as random walkers moving within the interstices of the extracellular space. The assumption of isotropic diffusion dictated a symmetrical probability distribution function for moving right or left along each axis $(x, y$, and $z$ ). The Monte Carlo simulator (see Fig. $3 A$ ) is compared against the analytical result for three-dimensional free diffusion from an instantaneous point source: 


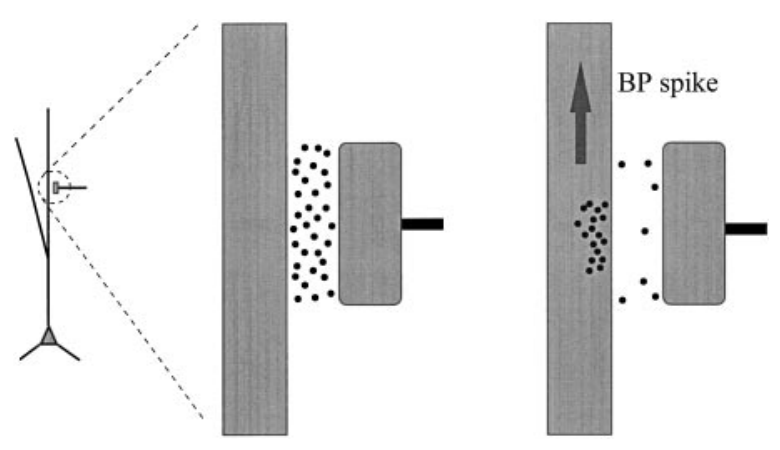

Figure 1. Back-propagating spikes are encoded as brief fluctuations in external calcium. Calcium (circles) in the synaptic cleft is shared. Experimental data suggest that a back-propagating spike travels relatively unattenuated along dendritic branches where A-type potassium currents have been inactivated. The occurrence of the back-propagating dendritic spike is associated with large influxes of calcium through voltage-gated channels. This influx is mirrored by a peri-dendritic efflux of calcium from the extracellular space. An overlying terminal would feel a decrement in the available calcium when a BP-spike (arrow) passes by.

$$
C(r, t)=\frac{N}{(4 \pi D t)^{3 / 2}} \exp \left(-r^{2} / 4 D t\right)
$$

where $C(r, t)$ is the expected concentration at radial distance $r$ at time $t$, $N$ is the number of molecules that started at the point source, and $D$ is the diffusion coefficient. Further tests covered a range of diffusion coefficients, as well as reflections from boundaries (data not shown). The Monte Carlo time step $(\theta=50 \mathrm{nsec})$ translates to a fixed step length (along each axis) of $\lambda=5.5 \mathrm{~nm}$ for $D=300 \mu \mathrm{m}^{2} / \mathrm{sec}$ and $\lambda=7.7 \mathrm{~nm}$ for $D=600 \mu \mathrm{m}^{2} / \mathrm{sec}$ (where $\lambda=\sqrt{ } 2 \mathrm{D} \theta$ ). The number of walkers released was between 5000 and $10^{6}$. With these parameters, the Monte Carlo calculations match the analytic results to within $<1 \%$. There are error bars in Figure $3 A$, but they are obscured by the size of the symbols.

After calibration against the analytic equations, we introduced cubic reflecting boundaries $0.806 \mu \mathrm{m}$ on a side. This side length yields the same volume as a sphere $1 \mu \mathrm{m}$ in diameter that approximates a synaptic bouton or spine head volume. Extended lengths of dendrites were made by concatenating multiple cubes into an elongated segment. Boundary elements were separated by small clefts of the same height as real synaptic clefts $(20 \mathrm{~nm})$. Typically, the total volume examined was a cube $7 \mu \mathrm{m}$ on a side. A minimum of $6 \times 10^{5}$ random walkers was placed at random in the interstices, the positions of all walkers were updated in parallel, and the walkers reflected off the boundaries after collision except when they collided with active calcium sinks on the boundary. After collision with an active calcium sink, the probability of a walker being consumed is defined by $P_{c}: P_{c}=0$ for inactive units and $0<P_{c} \leq$ 1.0 for consuming units.

Calcium extrusion is a first-order function of intracellular concentration, calibrated for a half-life between 35 and $500 \mathrm{msec}$ (Helmchen et al., 1996). For a half-life $t^{*}$, the probability of extrusion per walker per time step $\theta$ was:

$$
P_{\mathrm{ex}}=\left(1-P_{s}\right), \quad \text { where } \quad P_{s}^{t^{*} / \theta}=\frac{1}{2} \Rightarrow P_{s}=\mathrm{e}^{k \theta / t^{*}},
$$

where $k=\ln (1 / 2)$. Because sequestered molecules are extruded from their point of entry, this is equivalent to ignoring slow intracellular diff usion.

The finite-difference model. The model is illustrated in Figure 2. The basic cubic unit is called an intracellular unit (IU). IUs can be combined to represent spines, as well as longer segments that represent dendrites. These structures are modeled as reflecting boundaries except for the regions that represented active calcium sinks. The units are packed together tightly with an extracellular space (ECS) width of $20 \mathrm{~nm}$.

The extracellular space is subdivided into small parallelograms called ECS units. Each ECS unit holds a single-state variable that represents the average concentration in that volume. At each time step, an ECS unit updates its concentration as a function of its adjacent ECS neighbors and the calcium extruded by adjacent IUs. The IUs consume and extrude. For consumption of external calcium, an IU can either be in an active or

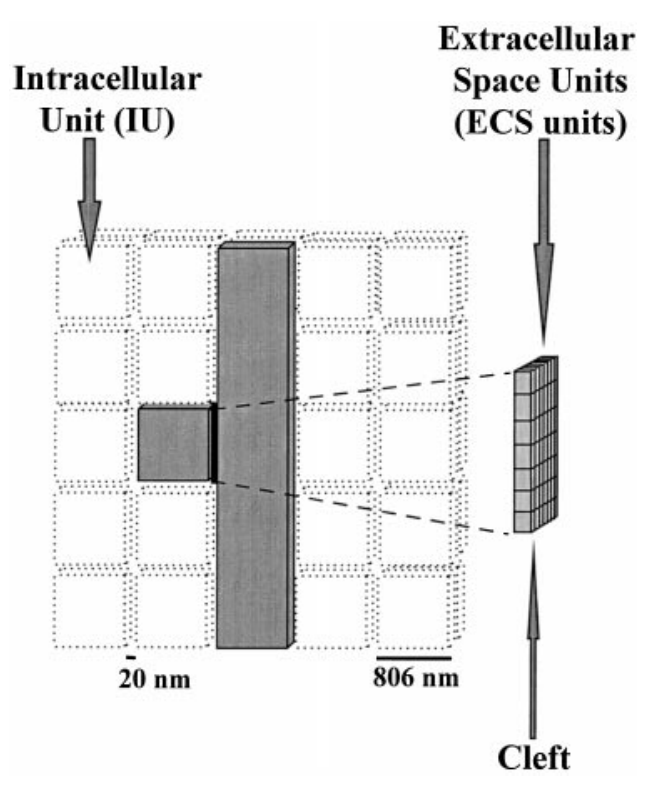

Figure 2. The finite-difference model. Elementary IUs are cubes 0.806 $\mu \mathrm{m}$ on a side (same volume as a sphere $1 \mu \mathrm{m}$ in diameter). IUs can be connected to form larger structures, such as dendrites and cell bodies. Shown in this figure are a presynaptic terminal (shaded cube) and a segment of dendrite (shaded rectangle). The clefts between the IUs are subdivided into smaller ECS units with side lengths of $115 \mathrm{~nm}$. Each ECS unit holds the average calcium concentration in that region. At each time step, each ECS subunit updates its concentration as a function of the concentration of its contiguous neighbors and as a function of the activity of the intracellular units with which it is in contact. The time step used is $2 \mu \mathrm{sec}$, which simulates diffusion with very little error at the specified spatial scales (see Fig. 3). In all studies (see Figs. 4-8), the dendritic segments are couched within a larger volume of simulated tissue, as indicated by the dotted cubes in this figure. The total volume is $5.8 \mu \mathrm{m}$ on a side, or $\sim 195 \mu \mathrm{m}^{3}$, which is sufficient to avoid border effects. The extracellular calcium concentration is initialized to $1.6 \mathrm{~mm}$ throughout the volume.

inactive state. Consumption takes place through some fraction of the surface of the IU that we generically call the consumption zone or calcium sink. The sinks can be spread evenly over the surface of an IU, or they can be clumped arbitrarily to represent uneven spatial distribution of calcium sinks. Consumption by an active calcium sink is described by a single parameter $P_{c} \in[0,1]$, which is engineered to correspond to the homologous parameter in the Monte Carlo calculations, above.

Although $P_{c}$ is a complicated function of channel distribution and open probabilities, we have made a simplifying approximation to represent calcium consumption during a dendritic action potential; a section of dendrite becomes active for a fixed amount of time and consumes a fixed number of external calcium atoms. In the studies presented below, we have used the fact that dendritic calcium channel densities are estimated between 1 and 15 channels $/ \mu \mathrm{m}^{2}$ (Magee and Johnston, 1995). If we estimate each channel to have a $10 \mathrm{pS}$ calcium conductance and a mean open time of $\sim 1 \mathrm{msec}$, then for a typical action potential, a density of 10 channels $/ \mu \mathrm{m}^{2}$ translates into a consumption of $\sim 21,500$ atoms $/ \mu \mathrm{m}^{2}$. Thus, for each cubic face of the model $\left(0.65 \mu \mathrm{m}^{2}\right)$, we have adjusted $P_{c}$ for an integrated consumption of 14,000 atoms/msec. Although the above calculation makes the rough assumption of a square-pulse $70 \mathrm{mV}$ depolarization, lasting for $1 \mathrm{msec}$, and further assumes that all the current is carried by $\mathrm{Ca}^{2+}$ atoms, the result is nonetheless consonant with other numbers from the literature. For example, 14,000 calcium atoms per active zone per spike has been estimated at a CNS terminal (the calyx of Held) (Helmchen et al., 1997); we have used this same value for total consumption at presynaptic terminals (see Figs. 4, 8). At a presynaptic terminal, 14,000 atoms/msec consumption would only require $\sim 13$ channels, which may seem surprising because $\sim 200$ calcium channels are thought to exist on a typical terminal (Smith, 1992). The apparent discrepancy is most likely explained by the possibility that most of the channels are not activatible when a spike arrives. To display explicitly the 

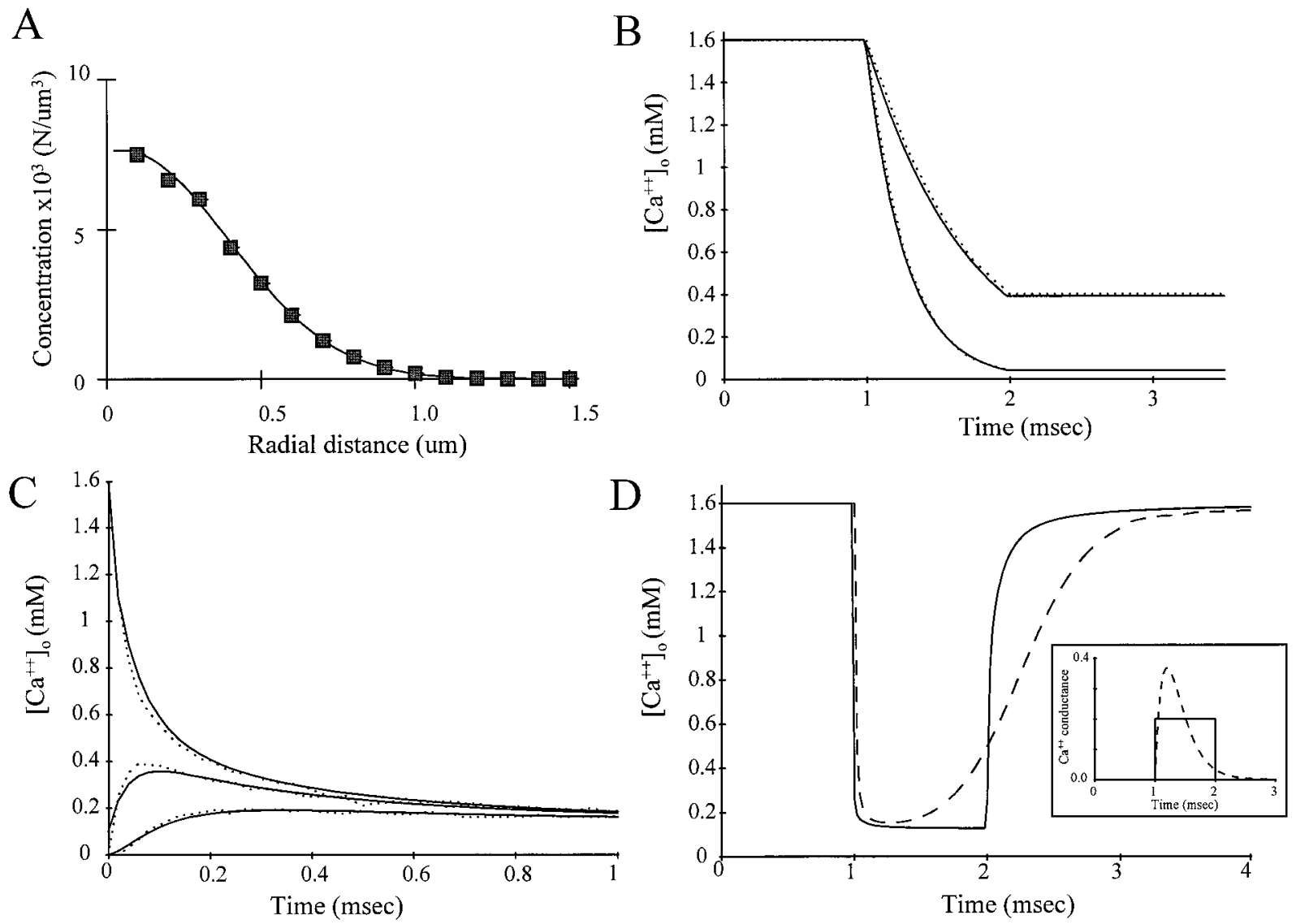

Figure 3. Comparison of the two models. $A$, Monte Carlo calibration to free diff usion. Five thousand random walkers were released in the middle of a volume with no barriers. The filled squares indicate the concentration of walkers in successive concentric spherical shells at $t=100 \mu$ sec and $D=600$ $\mu \mathrm{m}^{2} / \mathrm{sec}$, averaged over five runs. The solid line compares the analytically expected concentrations by the use of the three-dimensional free-diff usion equation (Eq. 1). B, Comparison of the function of the two models. Lateral diffusion is turned off so consumption can be assayed alone (i.e., the walkers are confined to a single cleft). Solid lines show the finite-difference model calculation for concentration in the cleft using two different values of $P_{c}: 0.00052$ (upper trace) and 0.00135 (lower trace). Dotted lines show Monte Carlo simulation under the same conditions with 5000 walkers. $C$, Measurement of diffusion without consumption. A single cleft is filled to resting levels (1.6 mM), whereas the rest of the ECS remains empty. The concentration is then measured as the walkers diff use from the original cleft into the neighboring extracellular space. Solid lines show the finite-difference model calculation for concentration in the cleft (upper trace), a neighboring cleft $0.8 \mu \mathrm{m}$ away (center-to-center distance; middle trace), and $1.6 \mu \mathrm{m}$ away (bottom trace). $D=600 \mu \mathrm{m}^{2} / \mathrm{sec}$. Dotted lines show the analogous Monte Carlo simulation with 10,000 walkers. As in all of our simulations, the basic IUs are $806 \mathrm{~nm}$ on a side, and the interstitial space between the IUs is $20 \mathrm{~nm}$. D, A $1 \mathrm{msec}$ square-pulse conductance change used to represent the effect of an action potential on voltage-gated calcium channels (solid line; inset) in simulations in this paper. For comparison, we show a conductance change represented by an $\alpha$ function, $\mathrm{f}(x)=x \cdot \exp (-x)$ (dashed line; inset). Under both profiles, the calcium fluctuations measured at a single $115 \times 115 \mathrm{~nm}$ active zone have approximately the same amplitude, whereas the concentration decrement is held longer when the $\alpha$ function is used. Thus, our use of square pulses here is conservative, because we are suggesting that time to recovery is an important functional variable.

dependence of our results on the total integrated current, we have explored consumption over a range of calcium channel density estimates (see Fig. 5). As will be shown, adjusting the density over a wide range will temper, but not eliminate, the computational ideas presented in this paper. That is, we are proposing that back-propagating action potentials can modulate the transmission probabilities of overlying presynaptic terminals; we will attempt to show that the modulation will hold true, even as the channel densities vary over a liberal range.

As part of our control, we compared our finite-difference model against Monte Carlo simulations in a number of conditions. Two examples are presented in Figure 3. In Figure 3B, the cleft is "sealed off" by prohibiting lateral diffusion outside the borders of the cleft, and consumption is then assayed at two different consumption probabilities $\left(P_{c}\right)$. In Figure $3 C$, a single cleft is filled with $1.6 \mathrm{~mm}$ calcium, whereas the neighboring extracellular space is set to zero concentration; the concentration is then measured in contiguous clefts as the calcium diffuses. In Figure $3, B$ and $C$, results from the two simulators match to within $1 \%$.

In the examples presented below (see Figs. 4-8), we modeled the conductance change in calcium channels as a square pulse (Fig. $3 D$, inset). The use of a potentially more realistic conductance change (Fig. 3D, inset, $\alpha$ function) yields results that are similar with respect to amplitude but that have a prolonged recovery time (Fig. $3 D$ ). Because we make statements in this paper about recovery time, we err on the conservative side by using square pulses.

The discrete dynamics for consumption are derived from a simple statistical argument; of a concentration of $C$ molecules in an ECS unit, the fraction of atoms within striking distance of the cell surface in the next time step is described by the ratio of the step length $\lambda=\sqrt{ } 2 \mathrm{D} \theta$ (where $\theta$ is the time step) to the cleft height $Z$. Of this fraction, half the atoms within reach will step toward the surface, whereas the other half will step away. Those that collide with the surface are absorbed with probability $P_{c}$ yielding:

$$
\Delta C=-\frac{1}{2} \frac{\lambda}{Z} C P_{c} .
$$

Extrusion takes place as a first-order function of intracellular concentration with a half-life $t^{*}$ :

$$
C_{\text {int }}(t)=C_{\text {int }}(0) e^{k t / t^{*}}
$$

where $k=\ln (1 / 2)$ and $C_{\text {int }}$ is the intracellular concentration underlying each ECS unit. Calcium is extruded back into the ECS unit from which 
it was consumed. Putting everything together, the concentration change in the $i$ th ECS unit $\Delta C_{i}$ is:

$$
\begin{aligned}
\Delta C_{i}=\text { fluxes }- \text { sinks }+ \text { sources; } \\
\Delta C_{i}=\frac{\tau}{\delta^{2}} D \sum_{j}\left(C_{j}-C_{i}\right) \\
-\left[2-\left(1-\frac{\lambda}{2 Z} P_{c}^{1}\right)^{\tau / \theta}+\left(1-\frac{\lambda}{2 Z} P_{c}^{2}\right)^{\tau / \theta}\right] C_{i}+\left(1-\mathrm{e}^{k \tau / t^{*}}\right) \\
{\left[C_{\mathrm{int}}^{1}+C_{\mathrm{int}}^{2}\right] . }
\end{aligned}
$$

$\tau=2 \mu$ sec is the time step of the finite-difference simulation, $\delta$ is the distance between the centers of the ECS units $(115 \mathrm{~nm}), D$ is the local diff usion coefficient, and $j$ sums over contiguous ECS neighbors (up to 12 in three dimensions). The second term represents depletion attributable to ion channels. $Z$ is the cleft height $(20 \mathrm{~nm})$, and $P_{c}^{1}$ and $P_{c}^{2}$ are the consumption probabilities (per Monte Carlo time step, $\theta=50 \mathrm{nsec}$ ) of the two IUs touching each ECS unit. $\lambda=\sqrt{ } 2 \mathrm{D} \theta$ is the average step length (along each Cartesian axis) of a random walker in time $\theta$. The choices for $\tau$ and $\delta$ were carefully calibrated against the Monte Carlo model.

A quick inspection shows that $\theta$ needs to be on the order of $50 \mathrm{nsec}$; otherwise, the step length of each walker would be close to or greater than the cleft height. In the finite-difference simulations, the time step $\tau=2 \mu \mathrm{sec}=40 \times 50 \mathrm{nsec}$; therefore, the second term is raised to an exponent $(\tau / \theta)$ or in this case the 40 th power. This latter maneuver allows us to account for the depletion that takes place in $\tau / \theta$ instances of $\theta$-size ticks. An example will illustrate. According to Equation 3, after a $\theta=50$ nsec time step, the new concentration in the cleft will be $C(t+\theta)=$ $\left[1-(\lambda / 2 Z) P_{c}\right] C(t)$. To represent the passage of $\tau=2 \mu \mathrm{sec}$ (finitedifference time step), we iterate the consumption $(\tau / \theta)$ times (in this case, 40 times), which yields a new cleft concentration of $C(t+\tau)=[1-$ $\left.(\lambda / 2 Z) P_{c}\right]^{\tau / \theta} C(\mathrm{t})$. The total change in cleft concentration after $\tau=2 \mu \mathrm{sec}$ is $\Delta C(t+\tau)=\left[1-\left[1-(\lambda / 2 Z) P_{c}\right]^{\tau / \theta}\right] C(t)$. The above representation of consumption compares within $1 \%$ error to Monte Carlo simulations (Fig. 3 ) and reduces computational time by four orders of magnitude.

Finally, the third term in Equation 5 represents first-order extrusion from the two IUs adjacent to the ECS unit.

As mentioned, all simulations were assayed between an upper bound $\left(D=600 \mu \mathrm{m}^{2} / \mathrm{sec}\right)$ and a lower bound $\left(D=300 \mu \mathrm{m}^{2} / \mathrm{sec}\right)$ for the diffusion coefficient. The diffusion of $\mathrm{Ca}^{2+}$ atoms in the networks of neural ECS will be slower than free diffusion, attributable at least to geometrical boundaries and buffering. The character and extent of extracellular calcium buffering are primarily unknown, but presumably the effect of ECS buffers will be absorbed in the local diffusion coefficient. There are currently no measures of "local" diff usion coefficients, but the past two decades have given us several studies of "long-distance" diffusion parameters in the mammalian brain. Experiments were pioneered in the early 1980s in which a current of test ion was injected in one location in the brain and the building concentration profile was measured at a distant site (30-200 $\mu \mathrm{m}$ away) (Nicholson, 1980; Nicholson and Phillips, 1981; Sykova, 1997). The results yield a dimensionless, empirical parameter called tortuosity, $\lambda$. Tortuosity relates the free-diffusion coefficient $D_{\text {free }}$ to the effective diffusion constant $D_{\text {eff }}$ :

$$
D_{\text {eff }}=\frac{D_{\text {free }}}{\lambda^{2}} .
$$

Under nonpathological conditions, $\lambda$ is within a narrow range of $1.5 \leq \lambda$ $\leq 1.7$. This range encompasses measurements in different species, over different parts of the brain, and made with cations (calcium, tetramethylammonium, and tetraethylammonium) or anions ( $\alpha$-naphthalene sulfonate and hexafluoroarsenate) (Nicholson, 1980; Nicholson and Phillips, 1981; Sykova, 1997). A value of $\lambda=1.6$ reduces $D_{\text {eff }}$ from $600 \mu \mathrm{m}^{2} / \mathrm{sec}$ in free solution to $234 \mu \mathrm{m}^{2} / \mathrm{sec}$ in the brain.

Such studies do not specify the diffusion coefficient locally. Because tortuosity involves paths through the bulk geometry, such a measurement is mute on the speed with which a molecule can move in an individual synaptic cleft. In separate studies, we have determined that the value of $\lambda$ inherent in our choice of geometry yields $\lambda=1.23$, which would reduce $D=600 \mu \mathrm{m}^{2} / \mathrm{sec}$ to $D_{\text {eff }}=395 \mu \mathrm{m}^{2} / \mathrm{sec}$ (D. M. Egelman and P. R. Montague, unpublished observations). Two possibilities, or a combination of the two, will account for the remaining slowing of diffusion measured in real tissue; (1) $D_{\text {local }}$ is $<600 \mu \mathrm{m}^{2} / \mathrm{sec}$, reflecting local extracellular binding, and/or (2) the extracellular space can be made more tortuous, by the combination of elementary units into larger units (such as somas), or equivalently some of the clefts can be clogged, acting as barriers to diffusion. Because there is no way to estimate accurately the contribution of these two possibilities, we run all our simulations over a wide parameter range, using $D_{\text {local }}$ values of 300 and $600 \mu \mathrm{m}^{2} / \mathrm{sec}$ as lower and upper bounds, respectively.

In summary, simulations used the following parameter values: diffusion coefficient $D=300-600 \mu \mathrm{m}^{2} / \mathrm{sec}$, side length of intracellular unit (cube) $=0.806 \mu \mathrm{m}$, size of smallest active zone $=115 \times 115 \mathrm{~nm}$, time step $=50 \mathrm{nsec}$ (Monte Carlo simulator) or $2 \mu \mathrm{sec}$ (finite-difference model), cleft height $=20 \mathrm{~nm}$, and the consumption parameter $P_{c}=$ $0.0005-1.0$.

\section{RESULTS}

\section{Back-propagating spikes can be encoded in peri- dendritic calcium fluctuations}

Back-propagating action potentials cause large fluxes of calcium into the dendrite; that influx is mirrored by an efflux of calcium from the peri-dendritic extracellular space. Figure $4 A$ shows a model presynaptic terminal directly contacting a dendritic shaft. During a back-propagating spike in the dendrite, the overlying terminal feels a large (38\%) drop in available external calcium. The known sensitivity of neurotransmitter release to external calcium (Dodge and Rahamimoff, 1967; Katz and Miledi, 1970; Mintz et al., 1995; Qian et al., 1997) suggests that such a decrement may influence the probability of synaptic transmission. If the presynaptic terminal were invaded by an action potential just after the passage of the back-propagating spike, the presynaptic release probabilities could be diminished. This effect highlights one of the important properties of external calcium signaling: the rapid bidirectional transfer of information at synaptic junctions. External calcium fluctuations encode information about postsynaptic activity (such as BP-spikes) as effectively as presynaptic activity (see Discussion).

\section{Addition of a dendritic spine with calcium sinks}

The geometry of the active dendritic elements influences the character of external calcium fluctuations. Figure $4 B$ shows a model presynaptic terminal contacting a dendritic spine. The spine consumes the same total calcium per unit area as the parent dendrite (see Materials and Methods). In this case, moving the synaptic junction away from the dendritic shaft (via a short spine) does little to change the calcium fluctuation experienced by the synaptic cleft. The situation changes significantly if the spine does not contain voltage-gated calcium channels or has them inhibited.

\section{Addition of a dendritic spine with inactive calcium sinks}

In Figure $4 C$, we consider the case in which voltage-gated calcium sinks on the dendritic spine are not active during the backpropagating spike, e.g., because of inhibition, inactivation, or absence. In this case, an $\sim 1 \mu \mathrm{m}$ inactive spine insulates the synaptic cleft from the large peri-dendritic calcium fluctuation demonstrated above. Thus, spine synapses could be distinguished from shaft synapses via fluctuations in external calcium felt by an overlying synaptic cleft.

\section{Density of calcium channels Extracellular signal}

As a control over the total integrated current, we have explored consumption over a range of calcium channel density estimates, changing the consumption parameter from 10 to $200 \%$ of our baseline estimate of $\sim 10$ channels $/ \mu \mathrm{m}^{2}$ (Fig. $5 A$ ). Even at onetenth of our baseline density, the concentration drops to 95.5- 

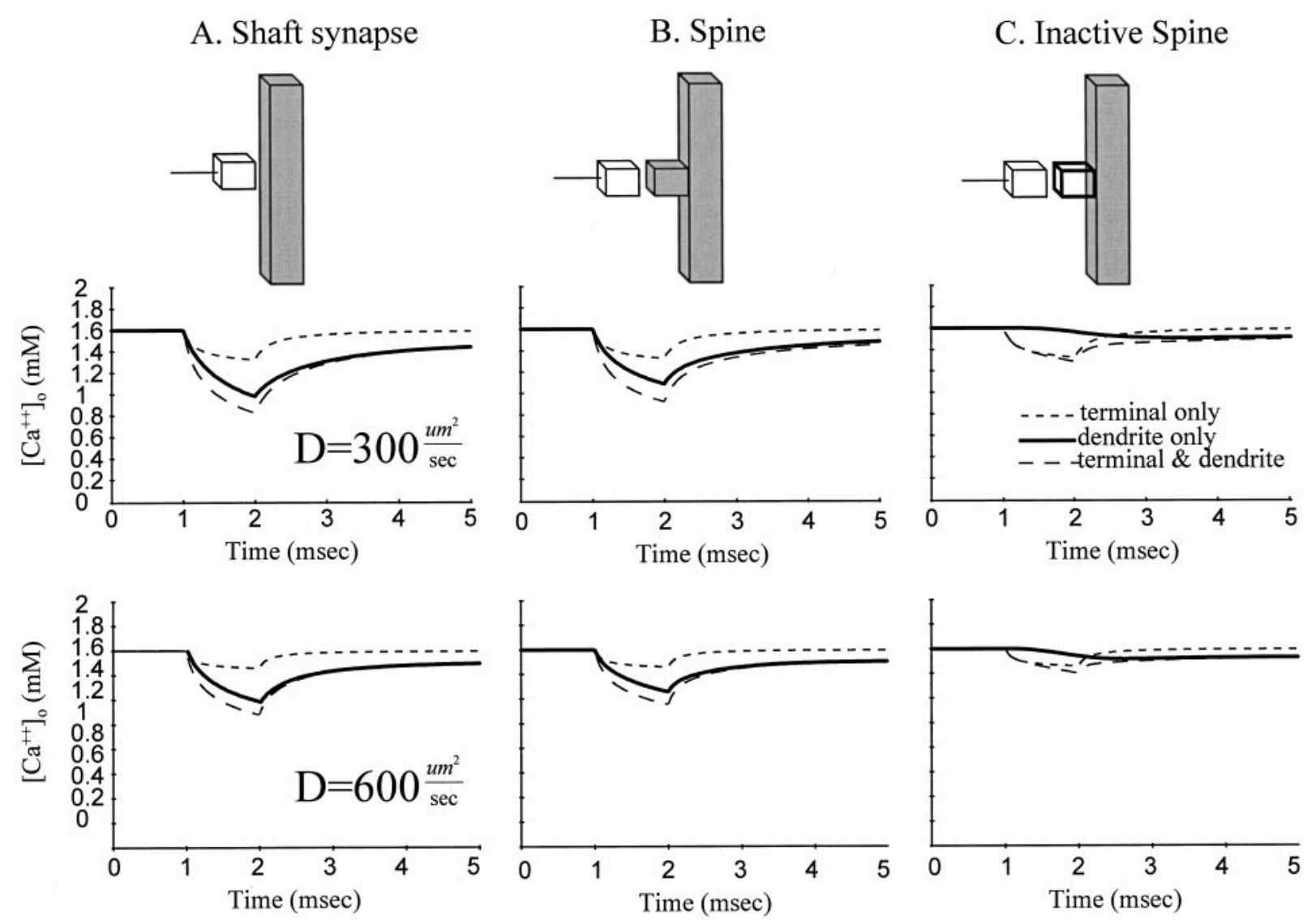

Figure 4. Large peri-dendritic calcium fluctuations may be reduced by moving the overlying terminal away. To examine the calcium fluctuations resulting from a dendritic action potential, we activated a $4.1 \mu \mathrm{m}$ dendritic segment by a back-propagating spike. The plots show the external calcium concentration in a synaptic cleft $(806 \times 806 \mathrm{~nm})$ shared between a presynaptic terminal and the dendrite (with or without a spine). Diff usion coefficients ( $D$ values) are between 300 and $600 \mu \mathrm{m}^{2} / \mathrm{sec}$, as shown in the middle and bottom rows. Calcium sinks are spread evenly along the dendrite. Three conditions are presented in each plot; (1) the terminal is activated by an invading action potential, (2) the dendrite is activated by a back-propagating action potential, or (3) the terminal and dendrite are activated simultaneously. The dendritic segments diagrammed in the top row are couched within a larger volume of simulated neural tissue, as in Figure 2. A, No spine. When the presynaptic terminal (open cube) directly contacts the dendritic shaft, the calcium in the cleft transiently drops between 27 and $34.5 \%\left(D, 600\right.$ and $300 \mu \mathrm{m}^{2} / \mathrm{sec}$, respectively) because of a back-propagating spike alone (solid trace). $B$, Addition of a spine. If the spine head and neck contain voltage-gated calcium conductances (VGCCs), the addition of a spine does little to change the external calcium fluctuation felt at the overlying terminal. The model spine here is an attached shaded cube $0.8 \mu \mathrm{m}$ on a side. Here the calcium in the cleft transiently drops between 22 and $32 \%\left(D, 600\right.$ and $300 \mu \mathrm{m}^{2} / \mathrm{sec}$, respectively) because of a back-propagating spike (solid trace); this is only slightly different from the result in $A$. $C$, Inhibition of a spine (attached open cube). If VGCCs on the spine do not become activated by a BP-spike (e.g., because of inhibition, inactivation, or absence), the geometry of the spine insulates the overlying bouton from the peri-dendritic calcium fluctuation. In $C$, a back-propagating spike causes a calcium decrement in the cleft of between only 5.4 and $6.1 \%\left(D, 600\right.$ and $300 \mu \mathrm{m}^{2} / \mathrm{sec}$, respectively), approximately fivefold smaller than in $A$ and $B$ above.

$96.8 \%$; a decrement of this magnitude will presumably translate into an $\sim 10 \%$ decrease in the probability of neurotransmitter release at an overlying terminal. At double the channel density, the concentration drops to $46.7-57.8 \%$, which represents an almost total depression of the release probability (Mintz et al., 1995; Qian et al., 1997). One of the central ideas in this paper is that back-propagating action potentials can modify the transmission probabilities of overlying presynaptic terminals. The details of this theme will become increasingly focused as more exact details about calcium channel kinetics, positions, and densities are discovered experimentally. However, we predict this idea will weather the test of new data, for calcium consumption will have effects even at one-tenth of our original estimate (corresponding to $\sim 1$ channel $/ \mu \mathrm{m}^{2}$ ).

\section{Intracellular signal}

One of the most important signals known in biology is calcium influx. To understand the relationship between the density of calcium channels and the resulting influx, we measure the total intracellular signal in our model. Figure $5 B$ shows that doubling the number of calcium channels on a surface will not suffice to double the influx. This is because taking more calcium from a cleft requires the sinks to wait for replenishment from diffusion from neighboring extracellular space. At a baseline density, total influx is between 12,243 and 12,581 atoms, which corresponds to a current of $\sim 4 \mathrm{pA}$ through a unit patch of membrane $\left(0.64 \mu \mathrm{m}^{2}\right)$. We will return to the issue of the intracellular signals below.

\section{Small-scale changes in calcium sink distribution have significant effects on calcium fluctuations}

In Figures 4 and 5, the calcium sinks were spread evenly over the dendrite. Figure 6 illustrates some of the consequences of putting calcium sinks in small clumps along the dendrite. To allow a comparison with the cases in Figure 4, we adjusted the parameters of the model so that the integrated calcium current per square micrometer was constant (see Materials and Methods). 
A

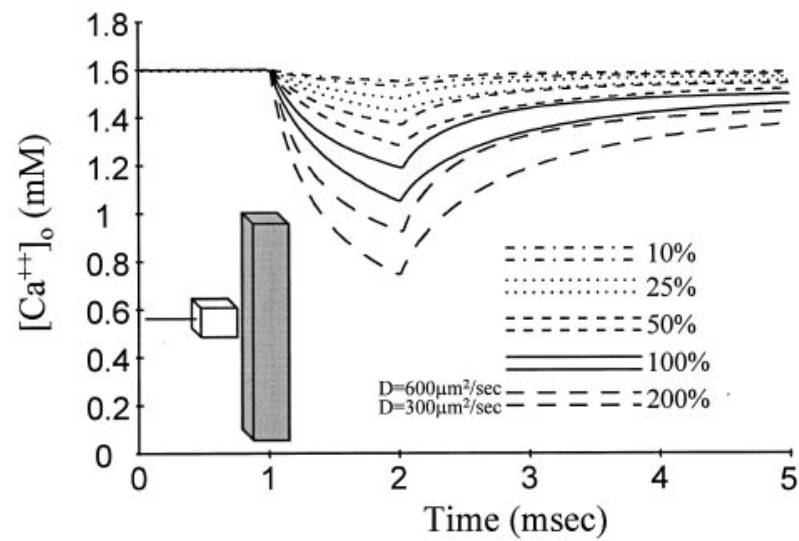

B

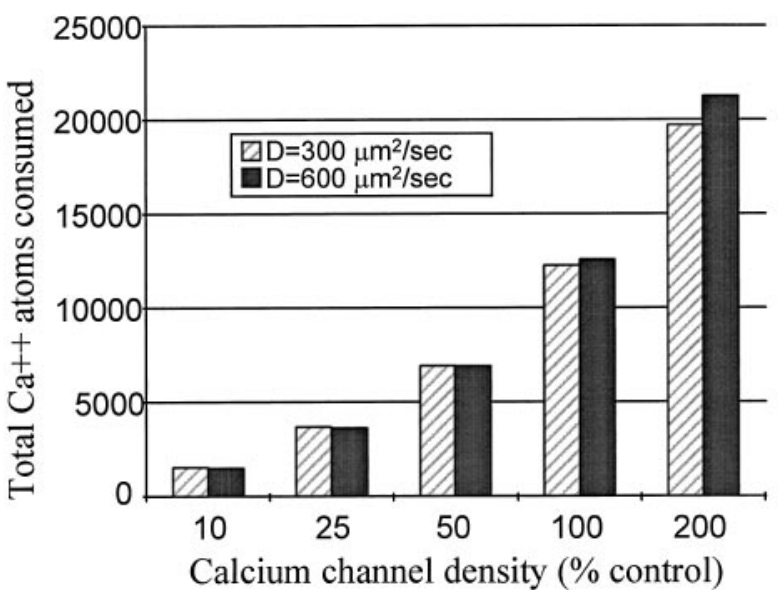

Figure 5. Changing the channel density affects extracellular and intracellular signals. $A$, Extracellular signal. The channel density covering the dendrite is changed from 10 to $200 \%$ of the values used in Figure 4. External calcium is measured in the cleft between the shaft and the overlying presynaptic terminal (inset). As always, the dendritic segment is couched within a larger volume of simulated neural tissue, as in Figure 2. Each calculation is bracketed by a diffusion coefficient $D$ of $300-600$ $\mu \mathrm{m}^{2} / \mathrm{sec}$ (lower and upper trace in each pair, respectively). $B$, Intracellular signal. Over a $806 \times 806 \mathrm{~nm}$ patch of membrane, the total calcium influx is a function of the channel density (modeled as consumption probability, see Materials and Methods). Doubling the density does not quite double the influx. The calcium depletion in the cleft does not fill in as rapidly in the $D=300 \mu \mathrm{m}^{2} / \mathrm{sec}$ case; thus at higher channel densities, the influx is lesser at 300 than at $600 \mathrm{\mu m}^{2} / \mathrm{sec}$. The difference at lower densities is artifactual.

With increasing clustering, local calcium fluctuations grow increasingly large. In the three cases examined, the time course of the average calcium concentration in the synaptic cleft was the same (Fig. 6D); however, the calcium immediately available to the consuming zones differs substantially (Fig. 6E).

\section{Coincident activation of neighboring dendrites extends calcium fluctuations in time and tissue space}

During development of the cerebral cortex, glial cells extend radial processes along which neuroblasts migrate. As a result, columnar regions of neuropil develop with vertical bundles of apical dendrites of pyramidal cells (Schmolke, 1987). It is unknown whether the dendritic bundles are leftovers of development or whether their existence is necessary for some computation. When viewed from the point of view of external calcium fluctuations, the bundle structure yields interesting possibilities.
In our previous examples, we examined the influence of one dendritic branch on calcium availability to synaptic clefts shared by overlying presynaptic terminals. In those examples, large decrements in local calcium levels recover quickly (within milliseconds) to baseline. This fact is easily understood, because in diffusion systems, replenishing fluxes are proportional to the concentration gradient and the concentration gradient is extremely large next to point sinks (presynaptic terminals) or even elongated line-like sinks (isolated active dendrite). However, a bundle of coactive neighboring dendrites will consume calcium from a spatially extended region, the gradients near the bundle center are small. Such synchronously active bundles cause a slower recovery of calcium near the center of the bundle (Fig. $7 A$ ). The recovery time has increased six- to eightfold, from 1 msec for an isolated dendrite (Fig. 4) to 6-8 msec (Fig. 7). The amplitude is also much larger than that in the comparable single dendrite case (Fig. 4).

The large fluctuations shown in Figure $7 A$ do not require precisely synchronous activity in the dendrites. Figure 7, $B$ and $C$, shows the calcium fluctuations in the center of the bundle and one unit away $(0.8 \mu \mathrm{m})$ from the bundle, respectively. In $B$ and $C$, each dendrite in the bundle makes a single spike but with Gaussian jitter in the exact time of the spike (mean time of spike production $=12 \mathrm{msec}$; SDs of 1, 5, and $10 \mathrm{msec}$ ). Even with large jitter, the bundle structure serves to increase the amplitude of the fluctuations in the center of the bundle (Fig. $7 B$ ). Interestingly, a reader measuring the calcium fluctuation from one unit away may have a hard time determining how synchronous the dendrites were; even with widely varying jitter, the calcium profiles look approximately the same from a short distance away (Fig. $7 C$ ).

\section{Magnitude of calcium influx may carry information about local geometry and activity}

Because intracellular enzymes carry different affinities for calcium, the exact size of a calcium influx signal can lead to diverse intracellular results (Bootman and Berridge, 1995). In Figure 8, we demonstrate how the local spatiotemporal surroundings can lead to different intracellular calcium signals, even given the same channels on a patch of membrane. In Figure $8 A$, we adjust the consumption parameter $P_{c}$ to yield an total influx of 14,000 at a presynaptic terminal, in keeping with results from Helmchen et al. (1997). This same $P_{c}$ is then used to define the consumption over an entire dendritic segment. The extracellular fluctuation is larger in the dendritic case, and the intracellular signal is correspondingly smaller. In this way the same patch of membrane can pass different signals to its intracellular signal depending on its local surroundings. Figure $8 B$ extends the studies of Figure 7 in which we examined the extracellular signals generated by asynchronous firing in a dendritic bundle. In Figure $8 B$, the magnitude of total calcium influx is assayed with different amounts of jitter in the firing times of the dendritic segments (SDs, 1-10 $\mathrm{msec}$ ). When all nine dendritic segments fire simultaneously, the influx across a given patch of membrane is approximately onehalf of its value at SDs $>10 \mathrm{msec}$. Thus, the magnitude of calcium influx carries information about the synchronicity of the bundle; as the bundle fires more synchronously, the total calcium influx is reduced.

\section{Dendritic activity convergence as an indexing mechanism}

The branched structure of dendrites in hippocampus and cortex creates an opportunity for dendrites from different (and distant) 

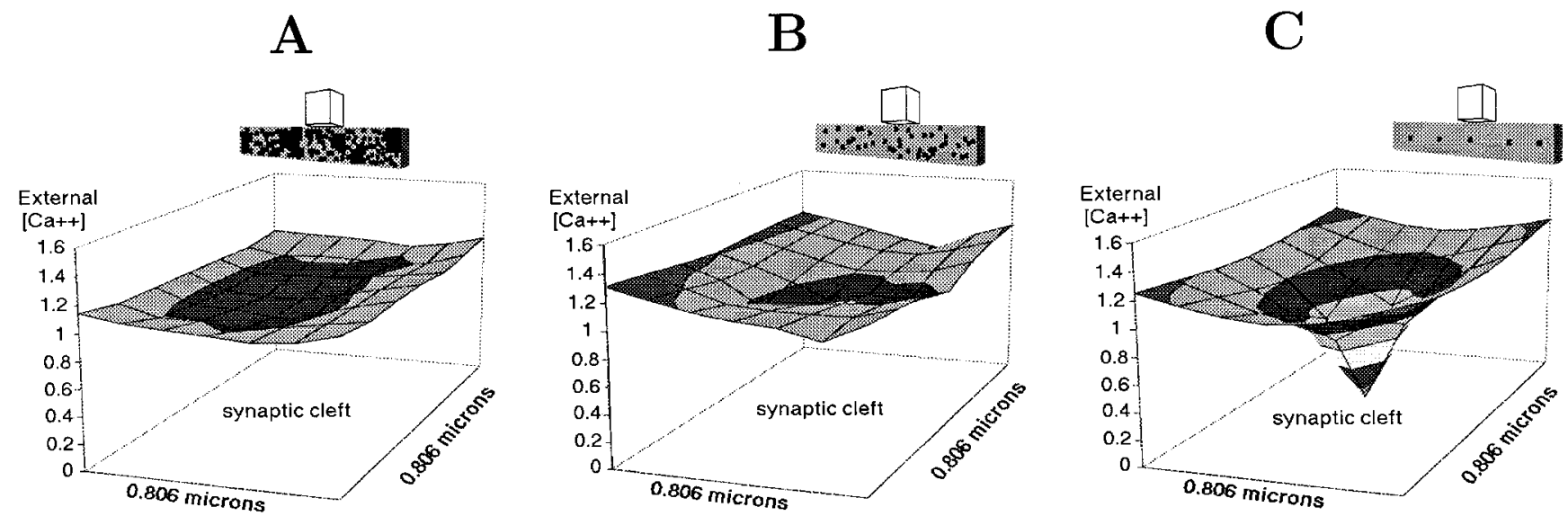

D average in cleft

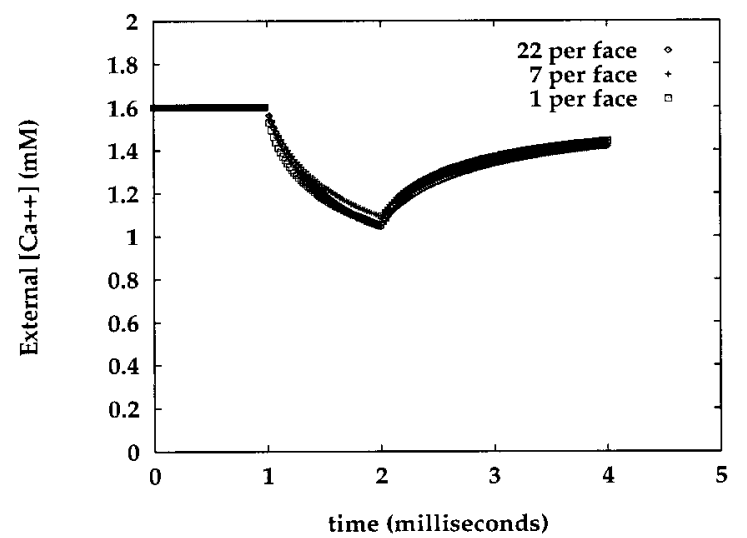

E available to cluster

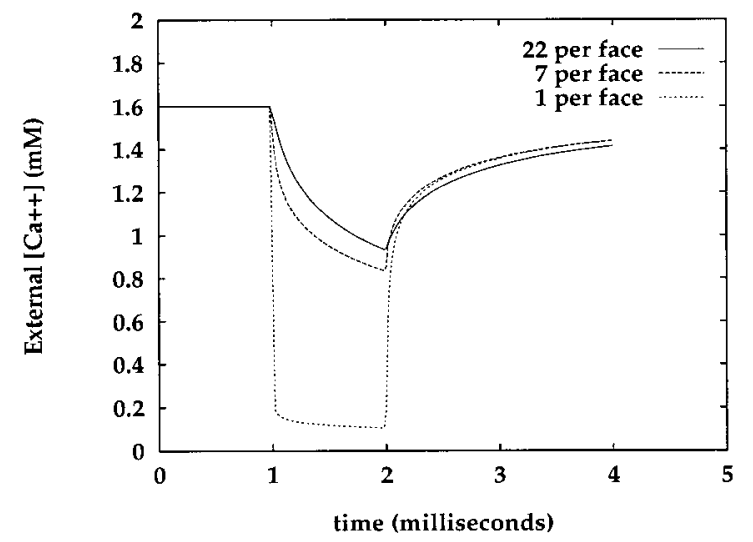

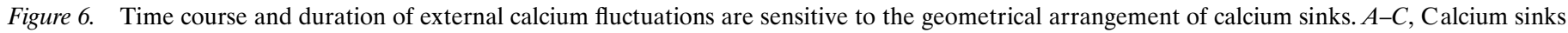

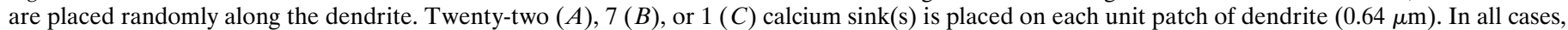

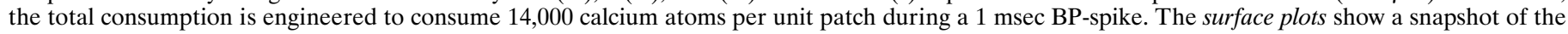

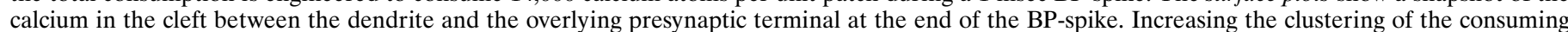

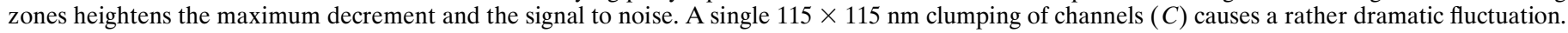

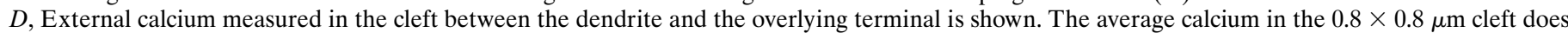

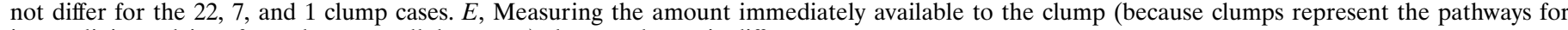
internalizing calcium from the extracellular space) shows a dramatic difference.

somas to come into contact as neighbors. In this way, a dendritic bundle-or meshwork-is achieved, in which back-propagating spikes may come together close in time and tissue space. Nearcoincident activity in these neighboring dendrites may engender the same sort of calcium signals as those examined in Figure 7.

We propose that external calcium fluctuations could be used in an addressing scheme in the brain. As indicated in Figure 9, such a mechanism could locate a specific volume of tissue by directing back-propagating spikes to that volume and by flagging the matching volumes with a large and temporally extended fluctuation in external calcium.

Recent physiological work on back-propagating spikes suggests one realistic possibility. It is known that back-propagating spikes tend to attenuate quickly because of the presence of A-type potassium channels in dendrites (Hoffman et al., 1997). This same work has shown that synaptic input inactivates these conduc- tances and causes dendritic spike propagation to proceed unattenuated into branches that have experienced the synaptic input. We propose here that this effect could mediate the addressing mechanism described in Figure 9: (1) coincident synaptic transmissions inactivate A-currents in dendrites present in a common volume of tissue; (2) back-propagating spikes are directed into the volumes in which transmissions occurred; and (3) large, temporally extended fluctuations in external calcium "mark" tissue volumes in which sufficient spatiotemporal overlap of backpropagating spikes occurs.

In short, high densities of synaptic input in a volume of neural tissue cause a convergence of dendritic spike activity, "calling" dendritic spikes into the volume in which the transmissions occurred. As illustrated in Figure 9, this mechanism permits widely broadcast activity, encoded in patterns of action potentials, to locate and flag specific volumes of tissue. 

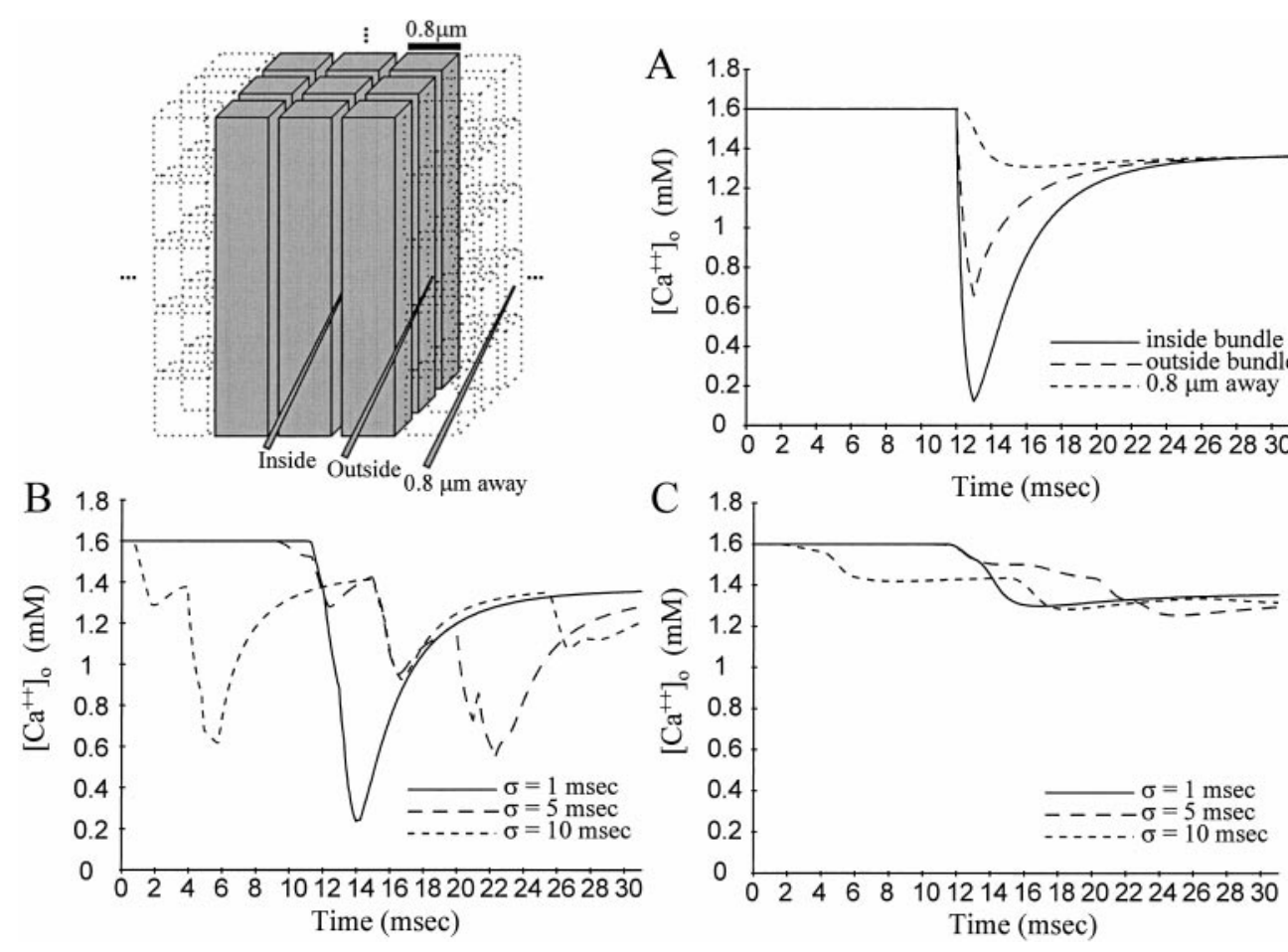

Figure 7. Synchronous spikes in a dendritic bundle scale the recovery time for external calcium. $A$, Peri-dendritic calcium fluctuations remain fairly local; i.e., their magnitude diminishes rapidly with distance through the tissue. However, different geometrical arrangements of these extended calcium sinks can dramatically change the time for external calcium to replenish by diffusion. Nine dendritic segments (each $4.03 \mu \mathrm{m}$ in length) are synchronously activated. The plot shows the external calcium measured in different locations relative to the bundle. The recovery time in the interior of the bundle has increased from $1 \mathrm{msec}$ in the single dendrite case to just under $10 \mathrm{msec}$. The large calcium decrement could block synaptic transmission for variable lengths of time. $B$, Synchronicity is not required for large calcium signaling. In this example, the activation time for each dendritic segment was drawn from a Gaussian distribution $($ mean $=12$ msec; $\sigma$ of 1,5 , and $10 \mathrm{msec}$ ). The external calcium measured in a cleft on the inner face of the bundle is shown. $C$, The same jitter in the activation times seen in $B$ is shown for external calcium measured in a cleft $0.8 \mu \mathrm{m}$ away from the bundle.

\section{DISCUSSION}

The implicit idea running through the examples in this paper is best cast as a question. If synapses require calcium to transmit, then why is all or most of the calcium pumped into the extracellular space, set to $1.5-2 \mathrm{~mm}$, and shared with neighboring neural and non-neural elements? Such an arrangement forces neighboring neural elements to share a resource that is necessary for neural transmission and general intracellular signaling but is in limited supply on short temporal and spatial scales. As we have demonstrated, resting levels of external calcium are not sufficiently high to protect against large fluctuations in this important resource. Instead, it seems as though the tissue is engineered so that external calcium levels are meant to fluctuate dramatically; given the functional importance of external calcium, we are led to the strong suspicion that external calcium fluctuations represent an information-bearing signal in the nervous system.

It is possible that some undiscovered compensatory mechanism protects neural tissue from the consequences of rapid external calcium fluctuations; however, many compensatory mechanisms are not plausible based on simple physical arguments. For example, any ionic channel permeable to calcium will cause the unidirectional movement of calcium out of the extracellular space because of the extreme calcium gradient established by cells; 1.5-2.0 mm outside and 50-100 nM inside translate into a 15,00040,000:1 outside-to-inside gradient. Thus, it seems unlikely that an ionic channel that fluxes calcium could counteract the expected fluctuations in external calcium. Calcium pumps and cotransporters will be unable to counterbalance the rapid fluctuations that we present in this paper, because they move calcium back into the extracellular space two to four orders of magnitude more slowly than the ionic channels steal it from the extracellular space.

\section{A bidirectional signal that may scale transmission probabilities}

In the synaptic cleft, one important event encoded in external calcium fluctuations is the occurrence of an action potential in either the dendrite or axon. As a signal, the calcium fluctuation is naturally fast and bidirectional, informing both pre- and postsynaptic elements of the arrival of a spike in the axon or dendrite. Moreover, for presynaptic spikes, an external calcium fluctuation does not depend on whether the terminal transmits. It is therefore possible that a rapid change in cleft calcium acts as a rapid anterograde and retrograde signal.

We have shown that even the firing of a single segment of dendrite can lower the external calcium drastically, and the decrement lasts a few milliseconds (Figs. 4-6). The computational function of dendritic spines has been long debated (Koch et al., 1992; Koch and Zador, 1993; Yuste and Tank, 1996); we propose here an additional role for spines. When the channels on a spine are inactivated, inhibited, or not present, the geometry of the spine is sufficient to protect the overlying presynaptic terminal from the large peri-dendritic calcium fluctuation caused by a back-propagating spike. We have also shown that when multiple, neighboring dendrites all propagate spikes, the local calcium fluctuation will be even larger and last longer (Fig. 7). Given the known sensitivity of neurotransmitter release on external calcium levels (Dodge and Rahamimoff, 1967; Katz and Miledi, 1970; Mintz et al., 1995; Qian et al., 1997), the demonstrated fluctuations may decrease the release probabilities of synaptic terminals nearby. This effect may implement a scaling of transmission probabilities that ranges from mild decrements to a full transmission failure.

\section{Transmission failures because of calcium fluctuations}

It is thought that a neuron conveys information in part through the pattern of action potentials it generates. This viewpoint is supported by recent experiments that demonstrate that mammalian neurons are capable of reliably producing spikes with a precision on the order of a millisecond. However, for the recipient neuron or tissue volume, i.e., the decoders of the message, the incoming spike train may not be the important variable; it may 

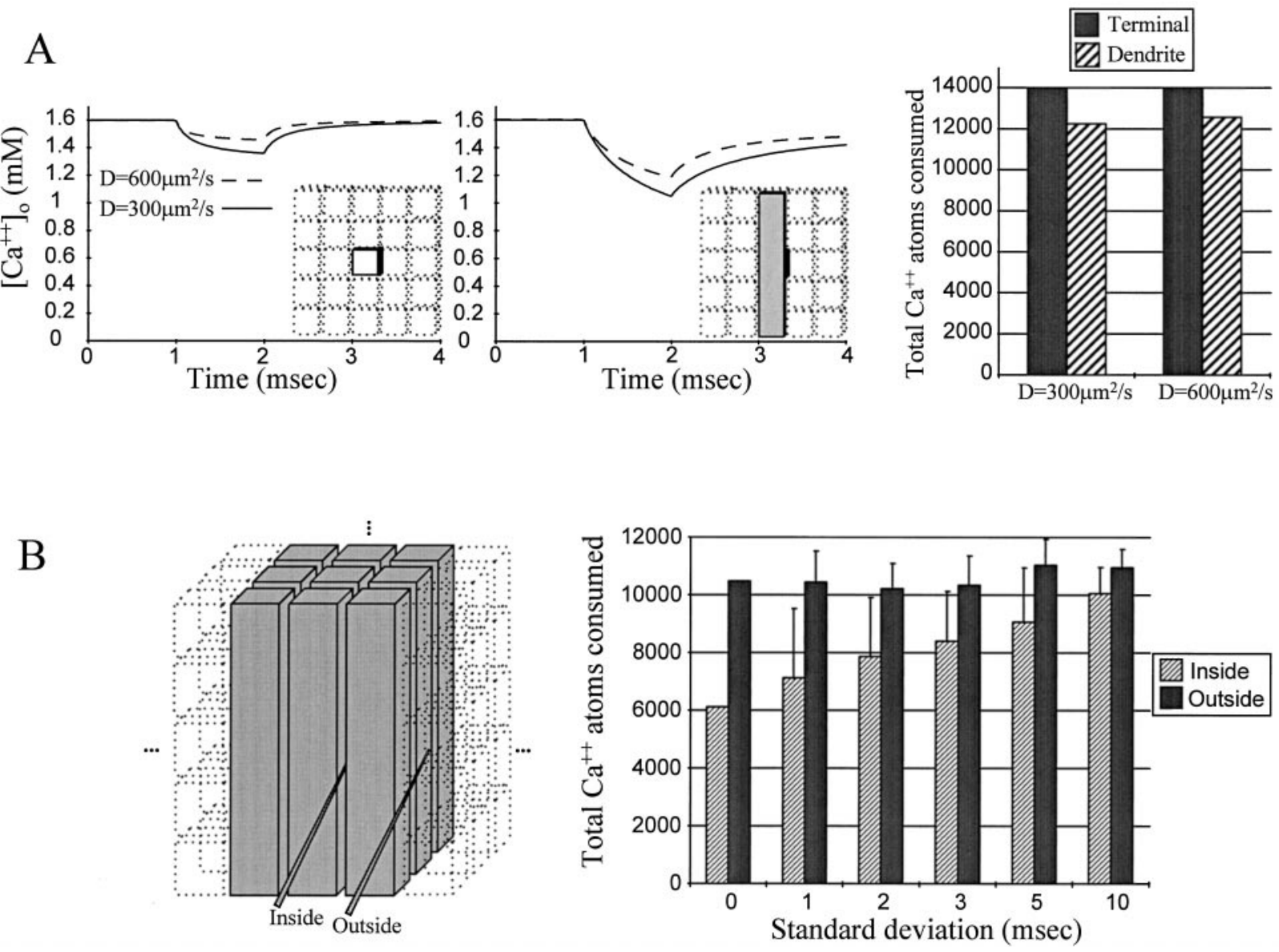

Figure 8. Magnitude of calcium influx can carry information about local geometry and synchronicity. Depending on the local spatiotemporal surroundings, a given set of calcium channels on a patch of membrane can lead to different extra- and intracellular calcium signals. A, Left, At a single presynaptic terminal, we adjust the consumption parameter $P_{c}$ to consume 14,000 ions during a 1 msec square-pulse action potential (Helmchen et al., 1997). This corresponds to a maximal change in the external calcium of $9-15 \%$. Middle, If that same value of $P_{c}$ (representing a certain concentration of calcium channels) is now used on the surface of a dendritic segment, the external calcium outside a unit patch of membrane $\left(0.64 \mu \mathrm{m}^{2}\right)$ now drops to $65.6-73.8 \%$. This larger change in external calcium is easily understood, because the calcium sinks now cover the surface of an entire dendritic segment, not simply a terminal face, and thus there is more total consumption. Right, The corresponding intracellular signal is $\sim 12 \%$ smaller in the case of the dendritic segment, because there is less external calcium available. $B$, A bundle of nine dendritic segments is asynchronously activated as shown in Figure 7, with SDs of 1, 2, 3, 5, and 10 msec. The same consumption parameter $P_{c}$ is used as in $A$. Calcium consumption at the outside surfaces of the bundle is approximately the same in all cases. However, on the inside surfaces, the magnitude of calcium influx carries information about synchronicity of the bundle; as the bundle fires more synchronously, the total calcium influx is reduced. For simplicity of illustration, we only show the results for $D=300 \mu \mathrm{m}^{2} / \mathrm{sec}$.

instead be the successful release of neurotransmitter. Synapses transmit only a fraction of the spikes that impinge on them, and recent work argues that mechanisms intrinsic to the presynaptic terminal make important decisions about whether a spike causes neurotransmission (Abbott et al., 1997). The point of view taken in this paper suggests that the postsynaptic neuron may also get to influence transmission at synaptic connections made on it.

By choosing when to generate a spike, a postsynaptic neuron can rapidly modulate the release probability of its overlying synaptic terminals via a decrement in extracellular calcium near its dendrites. In this manner, a spike in the recipient neuron that propagates into dendrites could block transmission at a connection that receives an axonal spike a short time later. This would eliminate transmissions caused by incoming spikes that the recipient neuron could, by some means, anticipate. In this scenario, transmission events at synapses occur when the postsynaptic neuron anticipates incorrectly the time of an incoming spike that would have otherwise caused a transmission; i.e., transmissions act somewhat like error signals.

\section{Noisy neurons and their bounded firing rates}

The transmission block would also place a limit on the maximal maintained firing rate of a recipient neuron. Cortical neurons tend to produce streams of spikes and silences at a temporal resolution of $\sim 1$ msec; i.e., firing rates above $1000 \mathrm{~Hz}$ are rare and not sustained for an appreciable number of spikes. With the idea of transmission blocks using external calcium fluctuations, a high spike rate traveling up the dendrites (e.g., $250 \mathrm{~Hz}$ ) could presumably block most afferent transmission. This would eliminate input drive to the recipient neuron, and spike production would decrease. As spike production drops, the number of transmission blocks drops, making it more likely that a transmission event will occur. Hence, the calcium depletion mechanism provides a means to keep 
A

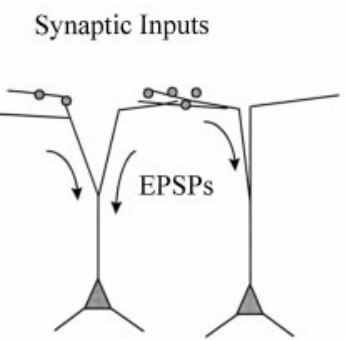

B

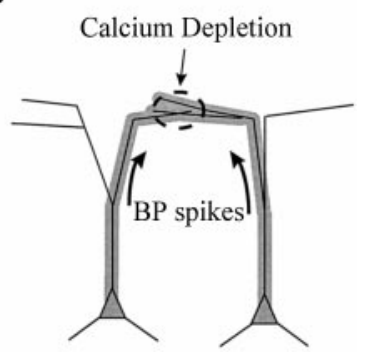

Figure 9. External calcium fluctuations are ideally suited to act as a marker signal for rapidly indexing a volume of neural tissue. $A$, In regions with a high density of synaptic transmission (filled circles), the EPSPs caused by the transmissions will deactivate A-type $\mathrm{K}^{+}$channels along that dendritic branch. $B$, Those same EPSPs may cause the soma to generate a spike, which will back-propagate (shaded lines) to the region of the high density transmission. In that region, the crossing of dendrites from different somas can lead to extended calcium decrements, of the type displayed in Figure 7. The calcium depletion zone indexes a specific region of the tissue.

a neuron noisy by bounding its spike production away from 0 , yet beneath a relatively well-defined maximal value.

\section{REFERENCES}

Abbott LF, Varela JA, Sen K, Nelson SB (1997) Synaptic depression and cortical gain control. Science 275:220-224.

Bootman M, Berridge M (1995) The elemental principles of calcium signaling. Cell 83:675-678.

Dodge FA, Rahamimoff R (1967) Co-operative action of calcium ions in transmitter release at the neuromuscular junction. J Physiol (Lond) 193:419-432.

Egelman DM, King RD, Montague PR (1998) Interaction of nitric oxide and external calcium fluctuations: a possible substrate for rapid information retrieval. In: Nitric oxide and other diffusible messengers in development, plasticity, and disease (Mize RR, Friedlander MJ, Dawson TJ, Dawson VM, eds). Elsevier Science.

Helmchen F, Imoto K, Sakmann B (1996) $\mathrm{Ca}^{2+}$ buffering and actionpotential-evoked $\mathrm{Ca}^{2+}$ signalling in dendrites of pyramidal neurons. Biophys J 70:1069-1081.

Helmchen F, Borst GG, Sakmann B (1997) Calcium dynamics associated with a single action potential in a CNS presynaptic terminal. Biophys J 72:1458-1471.

Hoffman D, Magee J, Colbert C, Johnston D (1997) $\mathrm{K}^{+}$channel regulation of signal propagation in dendrites of hippocampal pyramidal neurons. Nature 387:869-875.

Katz B, Miledi R (1970) Further study of the role of calcium in synaptic transmission. J Physiol (Lond) 789-801.

Koch C, Zador A (1993) The function of dendritic spines: devices subserving biochemical rather than electrical compartmentalization. J Neurosci 13:413-422.

Koch C, Zador A, Brown T (1992) Dendritic spines: convergence of theory and experiment. Science 256:973-974.

Magee J, Johnston D (1995) Characterization of single voltage-gated $\mathrm{Na}^{+}$and $\mathrm{Ca}^{2+}$ channels in apical dendrites of rat CA1 pyramidal cells. J Physiol (Lond) 487:67-90.

Mintz I, Sabatini B, Regehr W (1995) Calcium control of transmitter release at a central synapse. Neuron 15:675-688.

Montague PR (1996) The resource consumption principle: attention and memory in volumes of neural tissue. Proc Natl Acad Sci USA 93:3619-3623.

Nicholson C (1980) Modulation of extracellular calcium and its functional implications. Fed Proc 39:1519-1523.

Nicholson C, Phillips JM (1981) Ion diffusion modified by tortuosity and volume fraction in the extracellular microenvironment of the rat cerebellum. J Physiol (Lond) 321:225-257.

Nicholson C, tenBruggencate G, Stockle H, Steinberg R (1978) Calcium and potassium changes in extracellular microenvironments of cat cerebellar cortex. J Neurophysiol 41:1026-1039.

Qian J, Colmers WF, Saggau P (1997) Inhibition of synaptic transmission by neuropeptide $\mathrm{Y}$ in rat hippocampal area CA1: modulation of presynaptic calcium entry. J Neurosci 17:8169-8177.

Schmolke C (1987) Morphological organization of the neuropil in laminae II-V of rabbit visual cortex. Anat Embryol (Berl) 176:203-212.

Smith SJ (1992) Do astrocytes process neural information? In: Neuronalastrocytic interactions. Implications for normal and pathological CNS function (Yu ACH, Hertz L, Norenberg MD, Sykova E, Waxman SG, eds), pp 119-136. Elsevier Science.

Stuart G, Sakmann B (1994) Active propagation of somatic action potentials into neocortical pyramidal cell's dendrite. Nature 367:69-72.

Sykova E (1997) The extracellular space in the CNS: its regulation, volume and geometry in normal and pathological neuronal function. The Neuroscientist 3:28-41.

Yuste R, Tank D (1996) Dendritic integration in mammalian neurons, a century after Cajal. Neuron 16:701-716. 